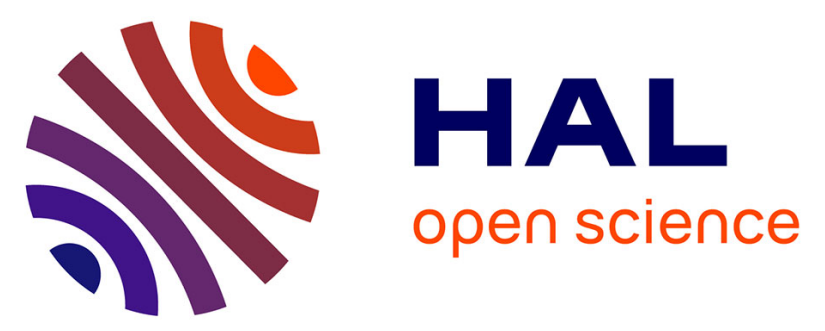

\title{
Extended persistence of antiphospholipid antibodies beyond the twelve-week time interval: Association with baseline antiphospholipid antibodies titres
}

Jean Devignes, Malika Smaïl-Tabbone, Alex Hervé, Geoffroy Cagninacci, Marie-Dominique Devignes, Thomas Lecompte, Stéphane Zuily, Denis Wahl

\section{To cite this version:}

Jean Devignes, Malika Smaïl-Tabbone, Alex Hervé, Geoffroy Cagninacci, Marie-Dominique Devignes, et al.. Extended persistence of antiphospholipid antibodies beyond the twelve-week time interval: Association with baseline antiphospholipid antibodies titres. International Journal of Laboratory Hematology, 2019, 41 (6), pp.726-730. 10.1111/ijlh.13094 . hal-02395258

\section{HAL Id: hal-02395258 \\ https://hal.inria.fr/hal-02395258}

Submitted on 6 Dec 2019

HAL is a multi-disciplinary open access archive for the deposit and dissemination of scientific research documents, whether they are published or not. The documents may come from teaching and research institutions in France or abroad, or from public or private research centers.
L'archive ouverte pluridisciplinaire HAL, est destinée au dépôt et à la diffusion de documents scientifiques de niveau recherche, publiés ou non, émanant des établissements d'enseignement et de recherche français ou étrangers, des laboratoires publics ou privés. 
11 pages, 2 tables, 1 figure

\section{Extended persistence of antiphospholipid antibodies beyond the twelve-week time interval: association with baseline antiphospholipid antibodies titres.}

Jean Devignes MD, $\mathrm{MSc}^{1}$, Malika Smaïl-Tabbone $\mathrm{PhD}^{2}$, Alex Hervé2 , Geoffroy Cagninacci Marie-Dominique Devignes $\mathrm{PhD}^{2,3}$, Thomas Lecompte MD, $\mathrm{MSc}^{4}$, Stéphane Zuily MD, $\mathrm{PhD}^{5,6}$ and Denis Wahl MD, $\mathrm{PhD}^{5,6}$.

${ }^{1}$ Haematology Laboratory, Nancy Academic Hospital, Vandœuvre-lès-Nancy, F-54511, France;

${ }^{2}$ Lorraine University, CNRS, Inria, LORIA, F-54000 Nancy, France;

${ }^{3}$ BioBase, Nancy Academic Hospital, Vandœuvre-lès-Nancy, F-54511, France;

${ }^{4}$ Geneva Platelet Group, Faculty of Medicine, University of Geneva; and Division of Angiology and Haemostasis, Geneva University Hospitals, Geneva, Switzerland;

${ }^{5}$ Division of Vascular Medicine, Regional Competence Centre for Rare Vascular and Systemic Autoimmune Diseases, Nancy Academic Hospital, Vandœuvre-lès-Nancy, F-54511, France;

${ }^{6}$ Lorraine University, Inserm UMR_S 1116, Vandœuvre-lès-Nancy, F-54511, France.

Corresponding author: Dr Jean Devignes,

Service Hématologie Biologique

Centre Hospitalier Régional Universitaire de Nancy

Rue du Morvan,

F-54511Vandœuvre-lès-Nancy Cedex, France

Phone: $33383153782 \quad$ Fax: 33383153796

j.devignes@chru-nancy.fr

Competing interest: the authors have no competing interests.

Running head: Extended aPL persistence beyond 12 weeks

Word count: 1817 


\section{Abstract \\ Introduction}

The confirmation time interval for presence of antiphospholipid antibodies (aPL) has been extended to 12 weeks since epiphenomenal antibodies may disappear after six weeks. Our aim was to analyse extended persistence of aPL positivity beyond the 12-week interval.

\section{Methods}

We retrospectively analysed our database of 23,856 aPL test samples collected between 2005 and 2017 from 17,367 consecutive patients. Two groups of patients were identified among aPLpositive patients, confirmed at 12 weeks: with or without extended persistence beyond confirmatory testing. Percentages of extended persistence are given according to the initial aPLpositivity profiles and baseline laboratory variables are compared between the two groups.

\section{Results}

Three hundred and twenty-seven patients confirmed aPL-positive had subsequent testing. The vast majority of them displayed extended persistence on the long term: $89.6 \%$ and up to $97.9 \%$ for patients with initial triple positivity. In extended persistent positive patients, there were more LA positive initial samples, and baseline LA test values and IgG aCL titres were higher than in non-persistent positive patients.

\section{Conclusion}

Data from a large database of an aPL referral laboratory showed that the time interval of 12 weeks defining persistence of aPL positivity was appropriate for the majority of patients. Furthermore, we found baseline features associated with extended persistence.

Keywords: Antiphospholipid antibodies, Antiphospholipid syndrome, Lupus anticoagulant, Anticardiolipin antibodies 


\section{Introduction}

According to the revised Sapporo classification for definite antiphospholipid syndrome (APS) [1], the classification criteria are met when at least one clinical criterion is present together with at least one antiphospholipid antibodies (aPL) positive test persisting over time. Current laboratory criteria are the lupus anticoagulant (LA), IgG or IgM anti-cardiolipin antibodies (aCL), or IgG or IgM anti- $\beta_{2}$-glycoprotein I antibodies $\left(\mathrm{a} \beta_{2} \mathrm{GPI}\right)$. The time interval defining persistence was extended from six [2] to 12 weeks [1] in 2006 because of concerns that epiphenomenal aPL -as in infections [3] - may disappear in some instances after more than six weeks. However, the 12-week interval was mostly based on expert opinion and little data are available about persistence of aPL positivity beyond this time frame. Indeed, some studies have questioned the relevance of using the 12-week instead of the six-week interval, but they included a limited number of subjects and lacked clear statistical significance $[4,5]$. Published data on confirmation of aPL positivity at 12 weeks were conducted in patients with APS. In particular, it was shown that most (53 out of 54) subjects with triple positivity at initial testing had their aPL profile confirmed after 12 weeks, whereas single-positive and double-positive groups had their positivity confirmed only for 21 out of 53 and 42 out of 50 subjects, respectively [6, 7]. However, no systematic study did analyse further testing beyond the confirmation at 12 weeks to assess persistence of aPL on the long term.

We retrospectively analysed our database derived from aPL laboratory testing in a real world setting of an aPL referral laboratory, with the main objective to evaluate the course of aPL positivity beyond confirmatory testing at 12 weeks; the secondary objective was to identify baseline features associated with such an extended persistence.

\section{Patients, material and methods}

Laboratory tests were performed according to established guidelines with the same tests and reagents for the LA and aCL tests throughout the study period (2005-2017), whereas the a $\beta_{2}$ GPI test was performed routinely from 2011 onwards. For LA testing, two screening coagulation tests 
were performed: dilute Russell's viper venom time (dRVVT LAC Screen ${ }^{\circledR}$, Siemens) and Activated Partial Thromboplastin Test $\left(\right.$ PTTLA $^{\circledR}$ Diagnostica Stago) [8]. All samples from patients anticoagulated with vitamin K antagonists (VKAs) that could not be tested with DRVVT have been tested with PTTLA, except for those on heparin treatment. For the two tests, positivity was defined by a LA ratio (clotting time of the patient sample relative to that of a locally prepared pool of plasmas obtained from healthy blood donors) greater than 1.2. In case of positive screening, confirmation tests were performed after 1:1 mixing with pooled plasma from healthy blood donors, using the same threshold ratio of 1.2. The LA test was deemed positive when at least one of the two LA ratios (PTTLA or dRVVT) was greater than the threshold, with mixing and confirmation tests alike.

Regarding aCL antibodies, IgG and IgM ELISAs (Enzyme-Linked ImmunoSorbent Assays) were performed according to guidelines applicable at the time of the testing [9]. IgG and $\operatorname{IgM} a \beta_{2} \mathrm{GPI}$ ELISAs were performed with a commercially available kit (Orgentec $\left.{ }^{\circledR}\right)$. For both ELISAs, the calibration was performed according to Harris's standards and the thresholds for positivity were defined as the $99^{\text {th }}$ percentile of the titre measured in a population of more than 170 healthy blood donors [10]. The cut-off values for aCL positivity were equal to 14 GPL and 5 MPL for IgG and IgM respectively, and those for $\mathrm{a}_{2}$ GPI positivity were equal to 8 GPL and 8 MPL for both IgG and $\operatorname{IgM}$.

Samples were deemed aPL-positive when at least one laboratory test was positive (above thresholds) and aPL-negative when all performed tests were negative (below thresholds).

A relational database was built to store, organize and query the data related to 23,856 samples from 17,367 consecutive patients visiting the Region Lorraine aPL referral centre at the Nancy University Hospital between 2005 and 2017. Data were directly imported from an extraction of records from our laboratory information system (GLIMS ${ }^{\mathrm{TM}}$; MIPS) into the database (MySQL system), thanks to a customized wrapper. SQL (Standard Query Language) procedures were developed on the database firstly to classify each sample as aPL-positive or not, depending on the results of the laboratory tests, and secondly to identify different subsets of patients according to the course of their aPL positivity. The underlying algorithm is displayed in Figure 1. The patient 
subgroup of interest was identified using five questions as (i) displaying an initial aPL-positive test with subsequent testing (questions $1 \& 2=$ yes), (ii) having their aPL positivity confirmed at an interval of at least 12 weeks (question 3 = yes; median time window: 26 weeks), and (iii) undergoing aPL testing thereafter (question 4 = yes). Finally (question 5), these patients were divided into two groups according to the persistence of positive aPL tests until the last studied sample (Extended Persistent Positive: EPP group), or not (Non-Persistently Positive: NPP group). EPP and NPP groups were compared regarding to qualitative data (counts with percentages) using the exact Fisher test. Quantitative data, calculated for initial positive tests only, were presented as median and interquartile range (IQR=Q3-Q1) because of the skewed distribution of the values and were compared using a non-parametric Wilcoxon test. A p-value $<0.05$ was considered significant.

\section{Results}

Course of aPL positivity beyond the 12-week time interval

To group patients according to their aPL positivity trajectories, the 23,856 sample results related to 17,367 consecutive patients (studied as per routine between 2005 and 2017) were computationally processed as described in Figure 1 . We found $82 \%$ of the patients negative for aPL $(n=14,617)$, and 2074 patients with a positive result that was not controlled at least 12 weeks apart. Out of the 676 remaining patients, $115(17 \%)$ were not found positive at the confirmatory testing and out of the 561 confirmed ones, 234 were not further tested. Thus, there were 327 aPLpositive patients, with confirmation at least 12 weeks apart and with subsequent testing. These patients have been followed up beyond confirmatory testing for a median duration of 94 weeks (IQR: 173, max: 393). Negative testing occurred for 34 out of the 327 patients during the followup period, yielding a percentage of extended persistence beyond confirmatory testing of $89.6 \%$.

\section{Extended persistence and initial aPL profiles}

Extended persistence was analysed with regard to initial aPL profile for a subgroup of 124 patients assayed with the three laboratory tests from 2011 onwards. Results are detailed in Table 1. In this 
group, the total percentage of extended persistence was $96.0 \%$ for a median follow-up duration of 56 weeks (IQR: 85, max: 278). Interestingly, this percentage was around 97\% for patients displaying double and triple positivity and slightly (but not significantly) lower (93.3\%) for those with single positivity. Among patients with initial single positivity, those with category IIa (LA only, 14 patients) were all extended persistent positive (EPP). In the double-positive group, the only non-persistent positive (NPP) patient had both LA and IgM aCL positivity at initial testing, and in the triple-positive group, the only NPP patient had the $\operatorname{IgG}$ isotype of aCL and combined $\operatorname{IgG}$ and $\operatorname{IgM}$ isotypes of $\mathrm{a} \beta_{2} \mathrm{GPI}$ at initial testing.

\section{Baseline features and extended persistence}

Comparisons of demographic and laboratory data between the 293 EPP and 34 NPP patients are presented in Table 2. The number of samples per patient for aPL testing was similar in the EPP and NPP groups (5 [IQR: 3] vs. 5 [IQR: 4] respectively). Laboratory results were analysed according to initial positivity. In comparison to the NPP group, there were significantly more LA positive samples in the EPP group, and baseline LA ratios and IgG aCL titres were significantly higher.

\section{Discussion}

In the revised Sapporo criteria [1], the time-interval for aPL confirmation was extended from six to 12 weeks on an empirical basis and expert opinion. Our retrospective study, conducted in a real world setting of an aPL referral laboratory, shows for the first time, that beyond the confirmation of aPL positivity at least 12-week apart, patients remain positive on subsequent testing in more than $89 \%$ of the cases up to 393 weeks of follow-up (median value: 94 weeks). Thus, these results fully support the choice that was made for the revised criteria. Whether a time-interval beyond six weeks but shorter than 12 is suitable remains to be studied in a prospective manner.

The subgroup of 124 patients tested with the three aPL tests enabled us to analyse extended persistence of aPL in relation with the initial aPL-positivity profile. Over their relatively short follow-up period (about one year on average), we observed very high percentages of extended 
persistence of aPL (> 93\%) regardless of initial single, double or triple positivity. Moreover, in the EPP group, we detected all three possible types of initial double positivity ( $\mathrm{aCL}$ and $\mathrm{a} \beta_{2} \mathrm{GPI}$, LA negative), (LA and aCL, a $\beta_{2}$ GPI negative) and (LA and $\mathrm{a} \beta_{2}$ GPI, aCL negative). The first one was the most frequent (19 out of 31) and corresponds to the only double positive combination described by Pengo and Denas in their recent review [7].

The complete dataset of 293 EPP and 34 NPP patients was large enough to study the baseline values of the LA and aCL tests associated with extended persistence. Patients found LA positive, and those with high IgG aCL titres or with high LA ratios (which can be considered as result of a high titre as well) at initial testing, were significantly more frequent in the EPP than in the NPP group. This extends existing reports concerning confirmation at 12 weeks, showing that presence of a LA is associated with aPL persistence at that time-point [6] and that patients with transient aPL-positivity have lower aCL titres than patients with confirmed aPL-positivity [10].

One limitation of our study is that it was a retrospective evaluation due to the real-world setting. However, such a setting ensured thorough exhaustiveness and reduced recruitment bias since all consecutive samples were analysed. Another limitation was that a 32 GPI antibodies were not tested routinely before 2011. Thus, aß2GPI single-positive samples before 2011 were classified as aPL negative. This could potentially lead to ignoring positive patients from our study or underestimating the percentage of extended persistence. However, this uncertainty does not contradict the conclusion of the paper.

\section{Conclusion}

We report what is to the best of our knowledge the first real-world evaluation of the 12-week time interval required for APS laboratory classification criteria, performed with a large sized dataset. Our data indicate that among aPL patients controlled after 12 weeks and confirmed positive, a vast majority remains persistently positive over time. Thus, the time frame of 12 weeks to control the initial aPL positivity seems appropriate to identify patients who will be persistently positive for aPL over the long run. Moreover, the database system and the analytical procedures developed 
for this study make it possible to expand our aPL patient registry in the future and consider further large-scale studies to improve the diagnosis, treatment and follow-up of these patients. 


\section{Acknowledgements}

Funding Support: We are grateful to Association des Chefs de Service for supporting AH. This work was funded by the Contrat de Plan Etat Région (IT2MP: Innovation Technologiques, Modélisation et Médecine Personnalisée) involving FEDER, Région Grand-Est, INSERM, Inria, CNRS and Université de Lorraine.

\section{Authors' contribution}

JD, MST, MDD, DW designed research. TL set up the aPL assays at the CHRU Nancy. JD, MST, MDD, AH performed research. GC built the database. JD, MST, MDD, AH performed statistical analysis. JD, MST, MDD, AH, TL, SZ and DW analysed the data. JD, MST, MDD, TL, SZ and DW wrote the paper. All authors approved the final manuscript. 


\section{References}

[1] Miyakis S, Lockshin MD, Atsumi T, Branch DW, Brey RL, Cervera R, Derksen RHWM, De Groot PG, Koike T, Meroni PL, Reber G, Shoenfeld Y, Tincani A, Vlachoyiannopoulos PG, Krilis SA. International consensus statement on an update of the classification criteria for definite antiphospholipid syndrome (APS). J. Thromb. Haemost. 2006; 4:295-306.

[2] Wilson WA, Gharavi AE, Koike T, Lockshin MD, Branch DW, Piette JC, Brey R, Derksen R, Harris EN, Hughes GR, Triplett DA, Khamashta MA. International consensus statement on preliminary classification criteria for definite antiphospholipid syndrome: report of an international workshop. Arthritis Rheum. 1999; 42:1309-1311.

[3] Abdel-Wahab N, Lopez-Olivo MA, Pinto-Patarroyo GP, Suarez-Almazor ME. Systematic review of case reports of antiphospholipid syndrome following infection. Lupus. 2016; 25 : $1520-1531$.

[4] Park SH, Jang S, Park C-J, Chi H-S. Clinical application of revised laboratory classification criteria for antiphospholipid antibody syndrome: is the follow-up interval of 12 weeks instead of 6 weeks significantly useful? Biomed Res Int. 2016; 2016:2641526.

[5] Solano C, Lamuño M, Vargas A, Amezcua-Guerra LM. Comparison of the 1999 Sapporo and 2006 revised criteria for the classification of the antiphospholipid syndrome. Clin. Exp. Rheumatol. 2009; 27: 914-919.

[6] Pengo V, Ruffatti A, Del Ross T, Tonello M, Cuffaro S, Hoxha A, Banzato A, Bison E, Denas G, Bracco A, Padayattil Jose S. Confirmation of initial antiphospholipid antibody positivity depends on the antiphospholipid antibody profile. J. Thromb. Haemost. 2013; 11: $1527-1531$.

[7] Pengo V, Denas G. Diagnostics and treatment of thrombotic antiphopholipid syndrome (APS): a personal perspective, Thrombosis Research 2018; 169: 35-40.

[8] Pengo V, Tripodi A, Reber G, Rand JH, Ortel TL, Galli M, De Groot PG. Update of the guidelines for lupus anticoagulant detection. Subcommittee on lupus anticoagulant/antiphospholipid antibody of the scientific and standardisation committee of 
the international society on thrombosis and haemostasis. J. Thromb. Haemost. 2009; 7: $1737-1740$.

[9] Lakos G, Favaloro EJ, Harris EN, Meroni PL, Tincani A, Wong RC, Pierangeli SS. International consensus guidelines on anticardiolipin and anti- $\beta 2$-glycoprotein I testing: report from the 13th International Congress on Antiphospholipid Antibodies. Arthritis Rheum. 2012; 64: 1-10.

[10] Wahl D, Devignes J, Mohamed S, Zuily S, Lecompte T. Definition and significance of high positivity aCL ELISA. Lupus. 2012; 21: 725-726. 
Table 1: Extended persistence of aPL beyond confirmatory testing at least 12 weeks apart, according to initial aPL positivity profile. EPP: Extended Persistent aPL-positive, NPP: Non-Persistent aPL-positive.

\begin{tabular}{|c|c|c|c|c|}
\hline Group of patients & $\begin{array}{c}\text { Total } \\
\text { number }\end{array}$ & EPP & NPP & $\begin{array}{c}\% \text { of extended } \\
\text { persistence }\end{array}$ \\
\hline $\begin{array}{l}\text { Subgroup tested for LA, aCL } \\
\text { and a } \beta 2 \text { GPI }\end{array}$ & 124 & 119 & 5 & $96.0 \%$ \\
\hline Single Positive & 45 & 42 & 3 & $93.3 \%$ \\
\hline Class IIa (LA) & 14 & 14 & 0 & \\
\hline Class IIb (aCL) & 18 & 16 & 2 & \\
\hline Class IIc (a $\left.\beta_{2} G P I\right)$ & 13 & 12 & 1 & \\
\hline Double Positive & 31 & 30 & 1 & $96.8 \%$ \\
\hline$a C L$ and $a \beta_{2} G P I$, LA negative & 19 & 19 & 0 & \\
\hline LA and $a C L, a \beta_{2}$ GPInegative & 7 & 6 & 1 & \\
\hline$L A$ and $a \beta_{2} G P I, a C L$ negative & 5 & 5 & 0 & \\
\hline Triple Positive & $48^{*}$ & 47 & 1 & $97.9 \%$ \\
\hline
\end{tabular}

*: Isotype distribution:

similar for aCL and IgG a $\beta_{2} \mathrm{GPI} ; 15 \mathrm{IgG}$ only; 4 IgM only; 10 both IgG and IgM isotypes; one $\mathrm{IgG} a \mathrm{aCL}$ and $\mathrm{IgM} a \beta_{2} \mathrm{GPI}, 8 \mathrm{IgG} \mathrm{aCL}$ and combined $\mathrm{IgG}$ and IgM isotypes for $\beta_{2} \mathrm{GPI}$ (including the NPP sample), $6 \mathrm{IgM}$ aCL and combined $\mathrm{IgG}$ and $\mathrm{IgM}$ isotypes for $\mathrm{a} \beta_{2} \mathrm{GPI}, 3$ combined IgG and IgM isotypes for $\mathrm{aCL}$ and $\operatorname{IgG} \mathrm{a} \beta_{2} \mathrm{GPI}$, and one combined IgG and IgM isotypes for aCL and $\operatorname{IgM} \mathrm{a} \beta_{2}$ GPI. 
Table 2: Characteristics of patients and baseline laboratory variables at the time of the initial positive testing according to extended persistence (EPP) or not (NPP) of aPL positivity beyond confirmatory testing.

\begin{tabular}{lcccc}
\hline Characteristics & $\begin{array}{c}\text { Total } \\
(\mathbf{n = 3 2 7})\end{array}$ & $\begin{array}{c}\text { EPP } \\
(\mathbf{n = 2 9 3})\end{array}$ & $\begin{array}{c}\text { NPP } \\
(\mathbf{n = 3 4})\end{array}$ & P value \\
\hline Demographic variables & & & & \\
\hline Age (years) & $45.3 \pm 16.4$ & $45.2 \pm 15.2$ & $46.2 \pm 17.8$ & 0.64 \\
Women & $242(74.0 \%)$ & $214(73.0 \%)$ & $28(82.4 \%)$ & 0.30 \\
\hline Laboratory variables & & & & \\
\hline LA positive & $208(63.6 \%)$ & $\mathbf{1 9 7}(\mathbf{6 7 . 2 \%})$ & $\mathbf{1 1}(\mathbf{3 2 . 4 \% )}$ & $\mathbf{0 . 0 0 0 1}$ \\
LA ratio (PTTLA) & $2.03[1.12]$ & $\mathbf{2 . 0 4}[\mathbf{1 . 1 3}]$ & $\mathbf{1 . 2 4}[\mathbf{0 . 0 6}]$ & $\mathbf{0 . 0 0 1}$ \\
LA ratio (dRVVT) & $1.51[0.57]$ & $\mathbf{1 . 5 3}[\mathbf{0 . 5 8}]$ & $\mathbf{1 . 3 5}[\mathbf{0 . 1 7}]$ & $\mathbf{0 . 0 2}$ \\
aCL positive & $206(63.0 \%)$ & $180(61.4 \%)$ & $26(76.5 \%)$ & 0.09 \\
IgG titre & $44[94]$ & $\mathbf{4 7}[\mathbf{9 2}]$ & $\mathbf{2 4}[\mathbf{1 0}]$ & $\mathbf{0 . 0 1 5}$ \\
IgM titre & $13[12]$ & $13[12]$ & $10[10]$ & 0.17 \\
\hline
\end{tabular}

The confirmatory testing was performed at least 12 weeks apart the initial one.

Qualitative data are presented as counts and percentages of corresponding group: $\mathrm{n}(\%)$. Except for age that is presented as mean \pm standard deviation, quantitative data are presented as median value and interquartile range [IQR] of positive tests: $\mathrm{m}$ [IQR] because of their skewed distribution. In bold are highlighted the differences between the PP and NPP groups that are statistically significant $(\mathrm{p}<0.05)$.

aCL: anticardiolipin antibodies, aCL positivity cut-offs were equal to 14 and 5 GPL and MPL for IgG and IgM respectively; LA: Lupus anticoagulant, LA positivity cut-off was a ratio $\geq 1.2$ for dRVVT or PTTLA screening tests (ratio of clotting time of the patient sample relative to that of a locally prepared pool of plasmas obtained from healthy blood donors). 
Figure 1: Flowchart of patient features according to the results of aPL testing.

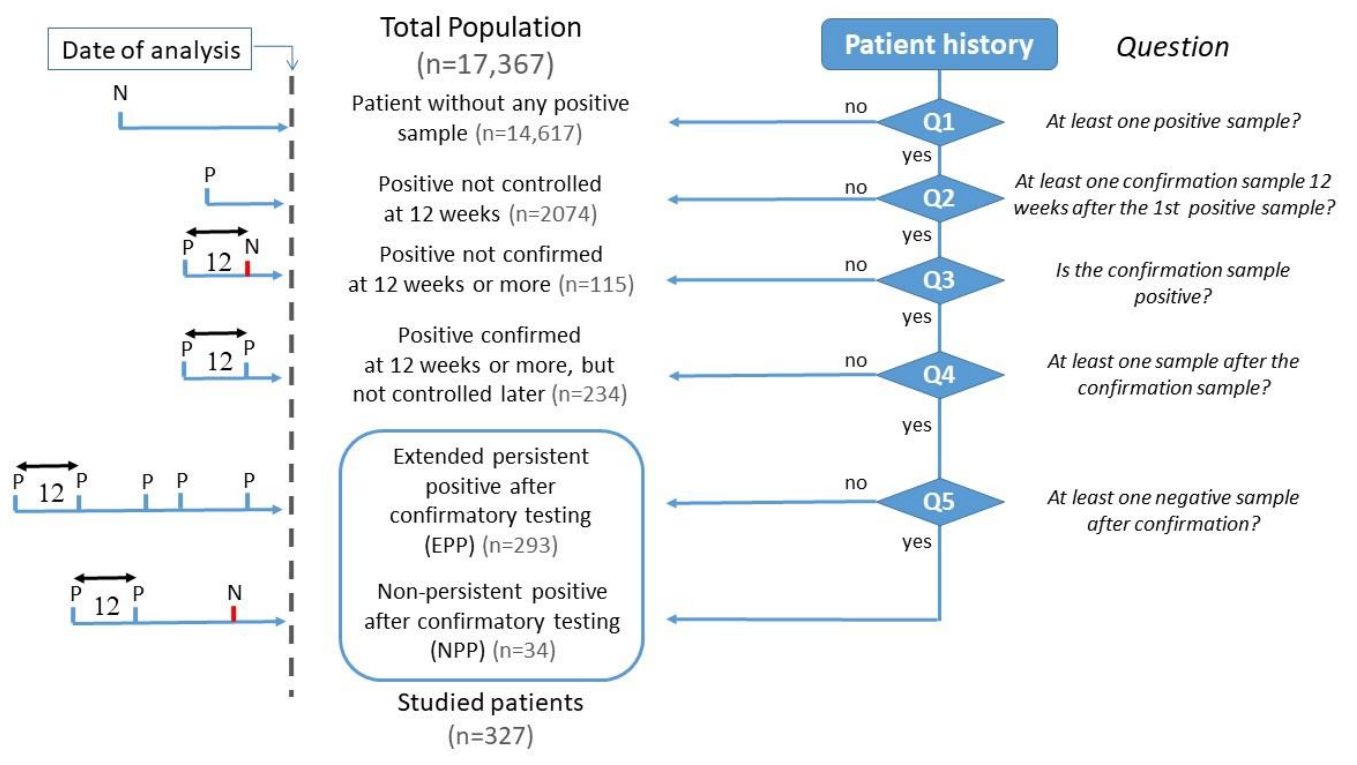

NB: This figure does not require colour processing. 